\title{
Bilateral Parapelvic Cyst Misdiagnosed as Hydronephrosis
}

\author{
Hong Sang Choi, Chang Seong Kim, Eun Hui Bae, Seong Kwon Ma, and Soo Wan Kim* \\ Department of Internal Medicine, Chonnam National University Medical School, Gwangju, Korea
}

A 36-year-old man visited our hospital with a suspected bilateral hydronephrosis that was detected during a routine check-up. Ultrasonography at the local clinic revealed a wide hypoechoic area in the center of both kidneys instead of a central echogenic complex (Fig. 1). Oliguria or abnormal laboratory values were not observed. On an axial view of the computed tomography with intravenous pyelogram (CT IVP) scan, low attenuated lesions with a cauliflower appearance were observed in the pelvises and calyces of both kidneys (Fig. 2A). No hydronephrosis or obstructive lesions in the urinary tract were observed on reconstructed intravenous pyelogram (IVP) image (Fig. 2B). A parapelvic cyst is a hypoechoic lesion located in the renal pelvis, which is often misdiagnosed as hydronephrosis because of its thin

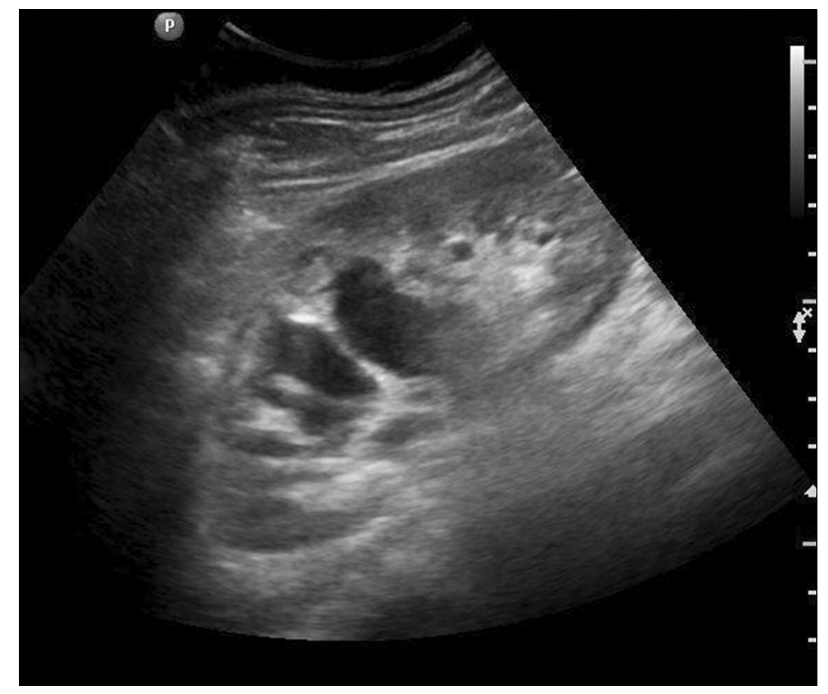

FiG. 1. Ultrasonography image of left kidney. Septa between the parapelvic cysts are well defined on ultrasonographic exam. wall. ${ }^{1,2}$ In conclusion, CT IVP is a useful test for the differential diagnosis of parapelvic cysts.

\section{CONFLICT OF INTEREST STATEMENT}

None declared.

\section{REFERENCES}

1. Koratala A, Alquadan KF. Parapelvic cysts mimicking hydronephrosis. Clin Case Rep 2018;6:760-1.

2. Tarzamni MK, Sobhani N, Nezami N, Ghiasi F. Bilateral parapelvic cysts that mimic hydronephrosis in two imaging modalities: a case report. Cases J 2008;1:161.
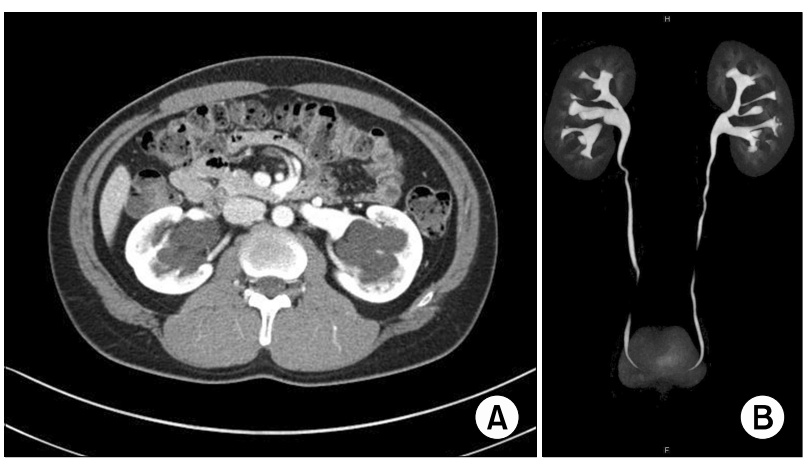

FIG. 2. Abdominal computed tomography scan with intravenous pyelogram (CT IVP) image. (A) Axial view of the CT IVP scan with contrast showing low attenuated lesions involving both the renal pelvis and calyces. (B) Urinary tract obstruction was not observed on reconstructed CT IVP image.

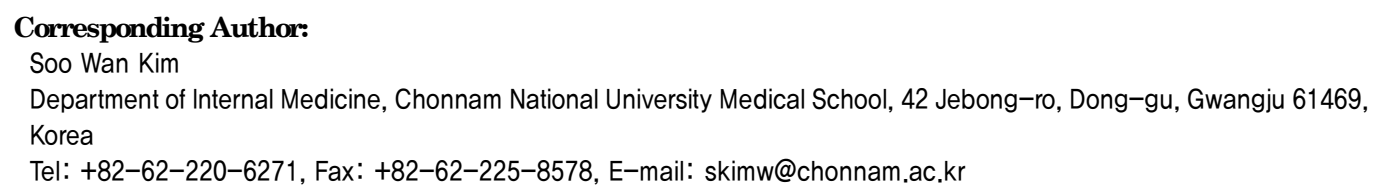

\section{Article History:}

Received September 27, 2018

Revised October 8, 2018

Accepted October 15, 2018

This is an Open Access article distributed under the terms of the Creative Commons Attribution Non-Commercial License (http://creativecommons.org/licenses/ by-nc/4.0) which permits unrestricted non-commercial use, distribution, and reproduction in any medium, provided the original work is properly cited. 